\title{
INFLUENCE OF ORAL ACYCLOVIR ON OCULAR COMPLICATIONS OF HERPES ZOSTER OPHTHALMICUS
}

\author{
G. W. AYLWARD, C. M. P. CLAOUÉ, R. J. MARSH and N. YASSEEM \\ London
}

\begin{abstract}
SUMMARY
The role of oral acyclovir ( $\mathrm{ACV}$ ) in the management of immunocompetent patients with herpes zoster ophthalmicus remains controversial. We have performed a retrospective, comparative, case-control study of cases seen in the Zoster Clinic at Moorfields Eye Hospital over the last 5 years. A standard proforma was used during this period to collect data on the rash, ocular involvement and treatment. There were 419 immunocompetent patients of whom 77 were treated with oral ACV prior to attending the clinic. We compared these with paired controls matched for age, sex and severity of rash. No difference in the rate of ocular complications between treated and untreated patients could be detected. This suggests that oral ACV as currently prescribed has little or no preventive effect on the ocular complications of ophthalmic zoster.
\end{abstract}

Zoster is due to reactivation from latency of varicellazoster virus, ' a member of the Herpetoviridae. Primary infection causes systemic disease (varicella), but reactivation causes a disease usually localised to a single dermatome (zoster). The virus which can be isolated from zoster vesicles is identical to that of varicella. ${ }^{2}$

Herpes zoster ophthalmicus (HZO) is associated with a high rate of ocular involvement, often resulting in serious morbidity. ${ }^{3}$ Most eye complications arise very shortly after the rash and are assumed to be induced by the presence of replicating varicella-zoster virions in the tissues. The majority of these complications are inflammatory: conjunctivitis, episcleritis, keratitis and uveitis. Conjunctivitis and episcleritis tend to be transitory and self-limiting, but the other inflammatory lesions can become chronic or recurrent, and can result in significant scarring of the corresponding tissue with loss of function. There is no incontrovertible evidence of replicating virus remaining in ocular tissues after the rash, but some have assumed its presence is associated with chronic inflammation. The lack of an animal model for HZO renders an understanding of the underlying pathophysiology difficult. ${ }^{+}$

Correspondence to: Mr G. W. Aylward, Bascom Palmer Eye Institute, PO Box 016880, Miami, FL 33101, USA.
Acyclovir (ACV) inhibits the replication of some members of the Herpetoviridae and is active against varicellazoster virus in vitro. ${ }^{5.6}$ The use of systemic ACV reduces the duration and spread of the rash and acute neuralgia in immunosuppressed patients, ${ }^{7}$ but its role in the immunocompetent remains controversial. ${ }^{8-10}$ Two prospective, controlled clinical trials have examined the effect of oral ACV on ocular complications of HZO, ${ }^{11-1.3}$ both of which reported a beneficial effect. We believe, however, that there are serious statistical and methodological flaws in both studies which cast doubt on this conclusion. Cobo et $a l .{ }^{12}$ reported a significant reduction in the rate of corneal complications and anterior uveitis in the ACV-treated group which they attributed to the effect of treatment. This would indeed be a valid conclusion if treated and control groups were similar. However, although randomisation was employed, the treatment and placebo groups were different in an important respect, in that there was a higher incidence of ocular involvement in the placebo group at the start of the study. The authors admit that the difference 'may introduce a bias to more ocular complications in the placebo group. ${ }^{12}$ The reported reduction in keratitis and anterior uveitis was not confirmed by the second study which used a higher dose of ACV. Of seven outcome measures investigated, the authors found a statistically significant difference for only one (the presence of active ocular disease 6 months after rash onset). ${ }^{13}$

In both studies a large number of outcome measures were investigated, which can introduce a subtle form of bias in favour of treatment. In order to claim a treatment effect, only one outcome measure has to be significantly different between the two groups at, say, the $p=0.05$ level. However, in order to reject a treatment effect, all of the outcome measures must be similar in each group. Hence the probability of either study finding no significant difference $(p=0.05)$ if no difference exists, is $0.95^{n}$, where $n$ is the number of outcome measures examined. In the first study ${ }^{11}$ the number was 18 , giving a probability of $0.95^{18}=0.4$, i.e. a less than evens chance that the study would accept the null hypothesis. This form of bias can be 
avoided either by deciding in advance how many outcome measures have to be different in order to claim a treatment effect, or by combining the outcome measures into a single score.

Doubts about the quality of the evidence for a beneficial effect of $\mathrm{ACV}$ on ocular complications in $\mathrm{HZO}$ prompted us to perform a comparative case-control study on a large number of patients with $\mathrm{HZO}$, specifically to detect an effect of oral ACV on ocular complications.

\section{PATIENTS AND METHODS}

A standard proforma has been used in the Zoster Clinic at Moorfields Eye Hospital for the past 5 years to collect data on all new patients referred with a diagnosis of HZO. The information recorded included name, age, sex, severity of skin rash, treatment with oral $\mathrm{ACV}$, and details of ocular involvement. The severity of the skin rash was graded at the first clinic visit on a 3-point scale. The dosage of oral $\mathrm{ACV}$ and the interval between the onset of the rash and first dose were recorded. Treatment was defined as 'adequate' if an oral dose of $800 \mathrm{mg}$, five times a day, was begun within 3 days of onset of the skin rash and taken for 7 days.

The presence or absence of the following ocular complications at the first or subsequent clinic visits was recorded: episcleritis, nodular episcleritis, corneal involvement, anterior uveitis. Corneal complications were subclassified as epithelial disease, stromal keratitis, mucous plaque keratitis, corneal oedema and neurotrophic keratitis. The use of topical steroid and topical ACV were also noted, and whether the patient was still receiving treatment for active ocular disease 6 months following the onset of HZO.

\section{Statistical Methods}

The subgroup of immunocompetent patients who had been given oral ACV were identified and control patients were selected from the remainder. Each control was matched for severity of rash, age and sex to one of the treated patients. A full match was achieved for grade of rash and sex, with the match for age being within 1 year for all cases. Where items of data were missing, the matched pair concerned was excluded from the corresponding analysis. The proportions of treated and control patients free of each ocular complication were analysed using techniques for matched pairs with a dichotomous outcome. ${ }^{14}$ McNemar's test for significant difference was followed by point and interval estimation of the simple difference between the two outcome proportions. There were four possible outcomes for each matched pair of case and control with respect to any of the outcomes (e.g. presence of keratitis): (a) neither patient had the outcome, $(b)$ the control patient had the outcome but the treated patient did not, $(c)$ the treated patient had the outcome but the control patient did not and $(d)$ both patients had the outcome. These data can be presented as in Table I, where the numbers of matched pairs in each category is represented by $a, b, c$ and $d$. The proportion of control patients free of the outcome (e.g. keratitis) is therefore

$$
p_{1}=(a+c) / n
$$

where $n$ is the number of pairs. The proportion of treated patients free of the outcome is

$$
p_{2}=(a+b) / n
$$

The simple difference is $p_{2}-p_{1}$, which has an expected value of zero in the absence of any treatment effect.

In order to combine all the outcome measures into a single variable, a total ocular involvement score (OIS) was constructed as the simple sum of the values for each outcome. A value of 2 was attached to the presence of each measure, with the exception of episcleritis which was assigned a value of 1 , reflecting its lesser clinical importance. Hence a patient with episcleritis, stromal keratitis and anterior uveitis would score 5. The distribution of this score was not normal, but the difference between the OIS of each member of a matched pair was normally distributed, confirmed using the Shapiro-Francia $W^{\prime}$ test. ${ }^{15}$ Statistical analysis of this quantity was therefore carried out using parametric analysis based on the Student $t$ distribution.

Separate analyses were carried out on a subgroup of matched pairs in which the treated patient had received 'adequate' treatment.

\section{RESULTS}

There were 419 immunocompetent patients referred to the Zoster Clinic with an acute episode of $\mathrm{HZO}$ over the 5 year period. The number of immunocompetent patients who had been treated with oral ACV was 77 (18\%), of which 42 patients $(10 \%)$ received 'adequate' treatment. The analyses that follow were therefore carried out on 77 matched pairs of treated and control patients (a total of 154 patients), and 42 pairs of 'adequately' treated patients and control patients (84 patients). The sex distribution was 34 men and 43 women, with an average age at presentation of 63 years (range 33-86 years). The prescribing doctor was the general practitioner in 66 cases $(86 \%)$, a referring ophthalmologist in 7 cases $(9 \%)$ and was not recorded in the remaining 4 cases $(5 \%)$. The distribution of the grade of severity of the rash is shown in Fig. 1.

In the results that follow, a positive difference indicates a higher proportion free of the outcome in the ACV-treated group (i.e. a beneficial effect of ACV). The proportions of patients free of any ocular involvement along with the results of statistical analysis are shown in Table II. A small difference of $6.5 \%$ is estimated but this is not statistically significant $(p=0.44)$. The proportions free of the various types of ocular involvement are shown in Table III, which also includes. the results for the status of treatment at 6 months. There is a suggestion of a beneficial effect of treatment on nodular episcleritis but the statistical significance of this difference is low $(p=0.07)$. The difference in OIS between treated patients and controls and the results of statistical analysis are shown in Table IV. There is a small negative effect of ACV which is not statistically significant $(p=0.46)$. 
Table I. $2 \times 2$ table with letters $a-d$ representing the number of matched pairs in each category (see text)

\begin{tabular}{lcc}
\hline & \multicolumn{2}{c}{ No acyclovir } \\
\cline { 2 - 3 } & No keratitis & Keratitis \\
\hline Acyclovir: & $a$ & $b$ \\
No keratitis & $c$ & $d$ \\
Keratitis & $c$ & \\
\hline
\end{tabular}

Table II. Presence or absence of ocular involvement (episcleritis, nodular episcleritis, keratitis, iritis) for all 77 matched pairs of treated and control patients

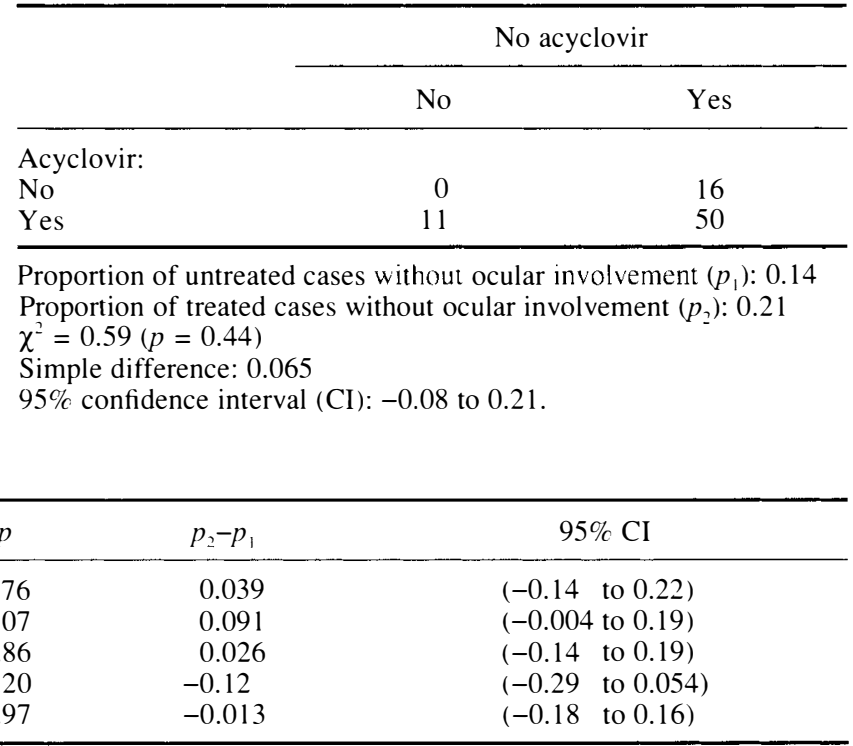

Table III. Proportions free of particular complications

\begin{tabular}{lcccccc}
\hline & $p_{1}$ & $p_{2}$ & $\chi^{2}$ & $p$ & $p_{2}-p_{1}$ & $95 \%$ CI \\
\hline Episcleritis & 0.62 & 0.66 & 0.098 & 0.76 & 0.039 & $(-0.14$ to 0.22$)$ \\
Nodular episcleritis & 0.88 & 0.97 & 3.3 & 0.07 & 0.091 & $(-0.004$ to 0.19$)$ \\
Corneal involvement & 0.48 & 0.51 & 0.028 & 0.86 & 0.026 & $(-0.14$ to 0.19$)$ \\
Anterior uveitis & 0.70 & 0.58 & 1.6 & 0.20 & -0.12 & $(-0.29$ to 0.054$)$ \\
On treatment at 6 months) & 0.45 & 0.44 & 0.001 & 0.97 & -0.013 & $(-0.18$ to 0.16$)$ \\
\hline
\end{tabular}

Table IV. Difference in ocular involvement score (OIS) between treated cases and controls

\begin{tabular}{lccc}
\hline Mean difference & $95 \% \mathrm{CI}$ & $t$ & $p$ \\
\hline-0.29 & $(-1.1$ to 0.49$)$ & -0.74 & 0.46 \\
\hline
\end{tabular}

Table V. Ocular involvement (episcleritis, nodular episcleritis, keratitis, iritis) for 42 matched pairs for which the treated patient received 'adequate' treatment

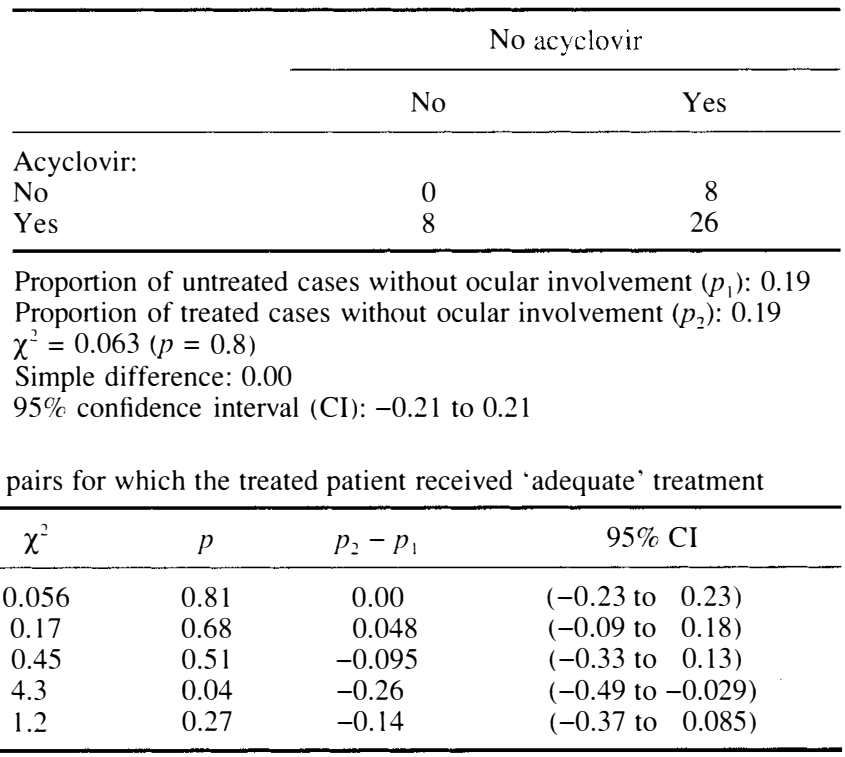

Table VI. Proportions free of particular complications for the 42 matched pairs for which the treated patient received 'adequate' treatment

\begin{tabular}{lccccccc}
\hline & $p_{1}$ & $p_{2}$ & $\chi^{2}$ & $p$ & $p_{2}-p_{1}$ & $95 \%$ CI \\
\hline Episcleritis & 0.63 & 0.63 & 0.056 & 0.81 & 0.00 & $(-0.23$ to 0.23$)$ & $(-0.09$ to 0.18$)$ \\
Nodular episcleritis & 0.90 & 0.95 & 0.17 & 0.68 & 0.048 & $(-0.33$ to 0.13$)$ \\
Corneal involvement & 0.50 & 0.40 & 0.45 & 0.51 & -0.095 & $(-0.49$ to -0.029$)$ \\
Anterior uveitis & 0.76 & 0.50 & 4.3 & 0.04 & -0.26 & $(-0.37$ to 0.085$)$ \\
On treatment at 6 months & 0.50 & 0.36 & 1.2 & 0.27 & -0.14 & $(-274)$ \\
\hline
\end{tabular}

Table VII. Difference in ocular involvement score (OIS) between treated cases and controls for 42 matched pairs for which the treated patient received 'adequate' treatment

\begin{tabular}{lccc}
\hline Mean difference & $95 \% \mathrm{CI}$ & $t$ & $p$ \\
\hline-0.69 & $(-1.7$ to 0.28$)$ & -1.4 & 0.15 \\
\hline
\end{tabular}

Table VIII. Difference in ocular involvement score (OIS) between treated cases and controls for various sub-groups of matched pairs

\begin{tabular}{lcccrr}
\hline Sub-group & $n$ & Mean difference & 95\% CI & $t$ & $p$ \\
\hline Rash grade 1 & 20 & -0.85 & $(-2.6$ to 0.86) & -1.0 & 0.32 \\
Rash grade 2 & 43 & -0.48 & $(-1.5$ to 0.52$)$ & -0.97 & 0.34 \\
Rash grade 3 & 14 & 1.1 & $(-1.0$ to 3.2) & 1.1 & 0.30 \\
Age up to 60 yr & 28 & -1.2 & $(-2.5$ to 0.094$)$ & -1.9 & 0.07 \\
Age 61-75 yr & 31 & 0.84 & $(-2.6$ to 2.0$)$ & 1.4 & 0.16 \\
Age 76 yr and over & 18 & -0.83 & $-0.98)$ & 0.36 \\
\hline
\end{tabular}




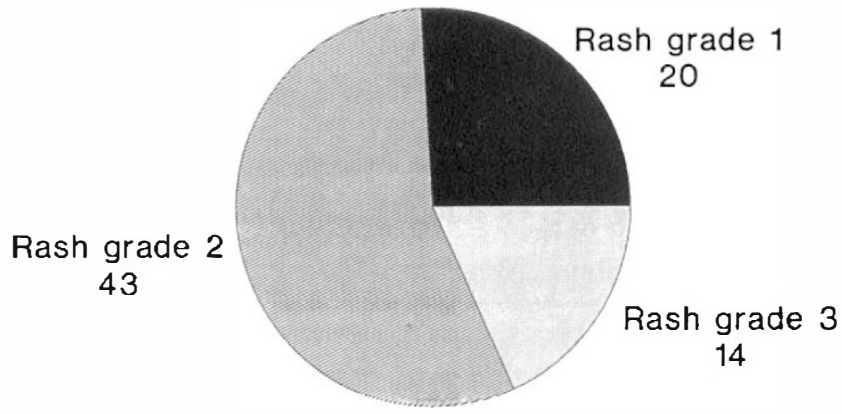

Fig. 1. Pie chart showing the distribution of grade of severity of rash among the 77 patients who received oral acyclovir.

Table X. Patients who received topical steroid

\begin{tabular}{lcr}
\hline & \multicolumn{2}{c}{ No acyclovir } \\
\cline { 2 - 3 } & Yes & No \\
\hline Acyclovir: & & \\
Yes & 38 & 16 \\
No & 17 & 4 \\
\hline
\end{tabular}

Proportion of untreated cases given topical steroid $\left(p_{1}\right): 0.73$

Proportion of treated cases given topical steroid $\left(p_{2}\right): 0.72$

$\chi^{2}=0.00(p=0.97)$

Simple difference: -0.013

95\% confidence interval: -0.17 to 0.15

A subgroup of matched pairs were considered in which the treated cases had 'adequate' treatment $(800 \mathrm{mg}$ five times a day for 7 days, begun within 3 days of the onset of the rash). There were 42 such pairs and the results are shown in Tables V, VI and VII. A negative effect of ACV on anterior uveitis is the only statistically significant result $\left.\left(p_{2}-p_{1}\right)=-0.26, p=0.04\right)$. Further analysis of the OIS was undertaken within subgroups broken down according to the grade of rash and three age bands (up to 60 years, 61-75 years, over 75 years). The results are shown in Table VIII. No statistically significant difference was found. In order to investigate the effect of the time interval between the onset of rash and the start of treatment, the 'adequately' treated group was divided into subgroups according to the number of days before treatment (Table IX). No significant difference was found.

The possible confounding effect of topical therapy was also examined (Tables X, XI). There was no significant difference in the proportion receiving topical steroid between treated and untreated groups $\left(p_{2}-p_{1}=-0.013\right.$, $p=0.97)$. Topical ACV was used more frequently in patients who had been prescribed oral ACV $\left(p_{2}-p_{1}=0.20, p=0.004\right)$.

\section{DISCUSSION}

A beneficial effect of oral ACV, as currently prescribed, on the ocular complications of HZO, was not detected by this study. Is it possible that a benefit exists but that the study failed to find it? The $95 \%$ confidence interval for the difference in OIS between the treated and untreated cases was -1.1 to 0.49 , and in the subgroup of cases with 'adequate' treatment was -1.4 to 0.28 . Therefore, even if the 'true' value of the difference was at the upper end of
Table IX. Difference in ocular involvement score (OIS) between treated cases and controls for sub-groups of matched pairs of 'adequately' treated patients according to the interval between onset of rash and start of treatment

\begin{tabular}{lrcccc}
\hline $\begin{array}{l}\text { Interval } \\
\text { days) }\end{array}$ & $n$ & Mean difference & $95 \%$ CI & $t$ & $p$ \\
\hline 0 & 5 & -1.8 & $(-4.8$ to 1.1$)$ & -1.2 & 0.31 \\
1 & 11 & -0.36 & $(-2.5$ to 1.8$)$ & -0.34 & 0.42 \\
2 & 12 & -0.75 & $(-2.6$ to 1.1$)$ & -0.82 & 0.32 \\
3 & 14 & -0.50 & $(-1.9$ to 0.90$)$ & -0.72 & 0.38 \\
\hline
\end{tabular}

Table XI. Patients who received topical acyclovir

\begin{tabular}{lcc}
\hline & \multicolumn{2}{c}{ No acyclovir } \\
\cline { 2 - 3 } & Yes & No \\
\hline Acyclovir: & 1 & 18 \\
Yes & 3 & 54 \\
No & & \\
\hline
\end{tabular}

Proportion of untreated cases given topical acyclovir $\left(p_{1}\right): 0.05$ Proportion of treated cases given topical acyclovir $\left(p_{2}\right): 0.25$

$\chi^{2}=9.33(p=0.004)$

Simple difference: 0.20

95\% confidence interval: 0.075 to 0.32

the first confidence interval, say 0.5 , then the clinical effect of ACV would be weak, accounting for an improvement of only half a point in a system by which episcleritis scores 1 . Thus, if a clinically significant effect of ACV exists, then it should have been detected by this study.

It is possible that ACV may have a different effect on some ocular complications compared with others. For example, the results suggest a beneficial effect on nodular episcleritis with a $9 \%$ difference between the two groups $(p=0.07)$. This result should be interpreted with caution, however, for two reasons. Firstly, the level of statistical significance is not high. Secondly, several $95 \%$ confidence intervals have been calculated, and as we described in the introduction, there is therefore a reasonable chance of a spurious result arising (only five $95 \%$ confidence intervals have to be calculated to make the chance of a spurious result nearly 1 in 4). This point also applies to the apparent negative effect of oral ACV on anterior uveitis in Table VI.

The use of topical therapy was not controlled for in this study, but has not confounded the results. The proportions of treated and untreated patients receiving topical steroid are virtually identical. This was not the case for topical $\mathrm{ACV}$, which was much more likely to be prescribed in patients given oral ACV. This difference would be expected to enhance a treatment effect rather than mask it, however.

The design of this study was retrospective, but that is not an intrinsic disadvantage. A common problem with a retrospective designs is missing data, but this was only a minor problem in the present study because the data were collected in a prospective fashion. An advantage of the retrospective design is the large number of patients available, which, combined with the use of matched pairs, 
gives high statistical power. The major disadvantage of this study, however, is the lack of randomisation which may have introduced bias. Is it possible that a beneficial effect of ACV is being obscured because it was prescribed for cases which already had or were more likely to develop ocular complications? We believe that this is unlikely for several reasons. Firstly, the ocular complications of zoster tend to appear several days after the $\operatorname{rash}^{16}$ and may not present until after ACV has been prescribed. Secondly, the vast majority of $\mathrm{ACV}$ treatment was initiated by the general practitioner, who is generally not equipped to diagnose ocular involvement. Thirdly, there is no evidence that any practitioner had a selective prescribing policy towards ophthalmic zoster, such that they prescribed ACV only for those cases with established ocular involvement.

The results of this study cast doubt on claims that oral $\mathrm{ACV}$ as currently prescribed reduces the incidence of ocular complications. It is of course possible that earlier administration of ACV may yield benefits. For example, a hastening of rash healing was not seen in patients who began therapy after 48 hours in a large multicentre study of non-ophthalmic zoster. ${ }^{17}$ The number of patients in our study treated early is small and the confidence intervals for treatment effect correspondingly large (Table IX). Therefore a treatment benefit from early treatment is compatible with our data. Unfortunately delays in presentation are likely to mean that early treatment is impractical in the majority of patients.

Key words: Acyclovir, Episcleritis, Herpes zoster ophthalmicus, Keratitis, Uveitis.

\section{REFERENCES}

1. Garland J. Varicella following exposure to herpes zoster. N Engl J Med 1943;228:336-7.
2. Caunt AE, Rondle CJM, Downie AW. Serological crossreactivity of the viruses of zoster and varicella. J Hygiene (Camb) 1961;59:249-51.

3. Womack LW, Liesegang TJ. Complications of herpes zoster ophthalmicus. Arch Ophthalmol 1983;101:42-5.

4. Easty DL. Ocular disease in varicella zoster infections. In: Easty DL, editor. Virus disease of the eye. London: LloydLuke, 1985:228-56.

5. Biron KK, Elion GB. In vitro susceptibility of varicellazoster virus to acyclovir. Antimicrob Agents Chemother 1980;18:443-7.

6. Furman PA. Chemotherapy of herpes virus infections. Curr Eye Res 1987;6:213-9.

7. Peterslund NA, Ipsen J, Schonheyder H, et al. Acyclovir in herpes zoster. Lancet 1981;2:827-30.

8. McKendrick MW, McGill JI, White JE, Wood MJ. Oral acyclovir in acute herpes zoster. BMJ 1986;293:1529-32.

9. McKendrick MW, McGill JI, Wood MJ. Lack of effect of acyclovir on postherpetic neuralgia. BMJ 1989;298:431.

10. Buchi ER, Herbort CP, Ruffieux C. Oral acyclovir in the treatment of ocular herpes zoster ophthalmicus. Am J Ophthalmol 1986;102:531-2.

11. Cobo LM, Foulks GN, Liesegang T, et al. Oral acyclovir in the therapy of acute herpes zoster ophthalmicus: an interim report. Ophthalmology 1985;92:1574-83.

12. Cobo LM, Foulks GN, Liesegang T, et al. Oral acyclovir in the treatment of acute herpes zoster ophthalmicus. Ophthalmology 1986;93:763-70.

13. Harding SP, Porter SM. Oral acyclovir in herpes zoster ophthalmicus. Curr Eye Res 1991;10(Suppl):177-82.

14. Fleiss JL. Statistical methods for rates and proportions. 2nd ed. New York: Wiley, 1981:113.

15. Altman DG. Practical statistics for medical research. London: Chapman and Hall, 1991:291.

16. Harding SP, Lipton JR, Wells JCD. Natural history of herpes zoster ophthalmicus: predictors of postherpetic neuralgia and ocular involvement. Br J Ophthalmol 1987;71:353-8.

17. Wood MJ, Ogan PH, McKendrick MW, et al. Efficacy of oral acyclovir treatment of acute herpes zoster. Am J Med 1988;85:79-83. 